\title{
Some Global Properties of Massless Free Fields
}

\author{
David E. Lerner $\star \star \star$ \\ Mathematical Institute, University of Oxford, Oxford 0X1 3LB, England \\ C. J. S. Clarke \\ Department of Mathematics, University of York, York Y01 5DD, England
}

\begin{abstract}
Elementary group-theoretical considerations show that global solutions to the massless free field equations are functions on the bundle of twistor dyads, rather than the bundle of conformal spin frames. Only in certain degenerate cases may they be thought of as ordinary spinor fields. This is the origin of the "Grgin discontinuity".
\end{abstract}

\section{Introduction}

It has been known for some time that global solutions to the massless free field equations

$$
\nabla^{A A^{\prime}} \varphi_{A^{\prime} B^{\prime} \ldots C^{\prime}}=0
$$

cannot generally be constructed on the conformal compactification of Minkowski space: if one attempts to extend a local solution to cover any closed null geodesic, a point is encountered at which the one-sided limits differ by a factor $i^{-2|s|-2}$, where $s$ is the helicity of the field. This phenomenon was first noticed by Grgin [1], and has recently been discussed by one of us [2] from the point of view of $U$-spinors. In this note, we show that the "Grgin discontinuity" arises from purely group-theoretical considerations - in particular from the fact that $\psi$ is required to be a density of conformal weight $-|s|-1$ in order that the field equations be invariant under $\mathrm{SU}(2,2)$. Given the correct transformation properties under the conformal group, the discontinuity will occur whether or not (1) is satisfied. Although global solutions to the field equations do exist for all values of $s$ [3], they cannot consistently be thought of as spinor fields in the usual sense.

This all turns out to be intimately related to the question of when a twistor dyad can be identified with a spin-frame, and this is the point of view from which we shall approach the question.

* Permanent address : Department of Mathematics, University of Kansas, Lawrence, Kansas 66045, USA

$\star \star$ Supported in part by the University of Kansas Research Fund and by the Science Research Council 


\section{Twistor Dyads and Representations of SU(2,2)}

The massless free fields of positive helicity can be conveniently realized as certain functions on the bundle of twistor dyads, the construction of which goes as follows (see [4] for more details):

Let $\mathscr{N}$ be the $0(2,4)$ null cone ${ }^{1}$, and regard $\mathbb{M}^{4}$ (Minkowski space) as a hyperplane section of this via the embedding

$$
\sigma(x)=\left(x, \frac{1}{2}\left[1+x_{\mu} x^{\mu}\right], \frac{1}{2}\left[1-x_{\mu} x^{\mu}\right]\right) .
$$

Let $\tilde{\mathscr{M}}:=\mathscr{N} / \mathbb{R}^{+}$be the rays of $\mathscr{N}$; this is a double covering of $\mathscr{M}$, the conformal compactification of Minkowski space. The isomorphism $P$ between simple twistor bivectors and points of the complexified null cone $\mathscr{C} \mathscr{N}$ is fixed by requiring that if $\left(U^{\alpha}\right)=\left(i x^{A A^{\prime}} \pi_{A^{\prime}}, \pi_{A^{\prime}}\right)$ and $\left(V^{\alpha}\right)=\left(i x^{A A^{\prime}} \omega_{A^{\prime}}, \omega_{A^{\prime}}\right)$ are two twistors through $x[6]$, then

$$
P: U \wedge V \rightarrow\left(\pi_{A^{\prime}} \omega^{A^{\prime}}\right) \sigma(x) .
$$

Let $\mathscr{S}^{*}:=\left\{(U, V): U \neq z V, z \in \mathbb{C}^{*}\right\}$, and define $\pi: \mathscr{S}^{*} \rightarrow \mathscr{C} \mathscr{N}$ by $\pi(U, V)=P(U \wedge V)$. Then $\mathscr{S}:=\pi^{-1}(\mathscr{N})$ is called the bundle of $t$ wistor dyads over $\mathscr{N}$. It can be made into a principal $\operatorname{SL}(2, \mathbb{C})$ bundle by defining

$$
W_{0^{\prime}}=U, W_{1^{\prime}}=V
$$

and

$$
W_{A^{\prime}} \cdot \beta=W_{B^{\prime}} \bar{\beta}_{A^{\prime}}^{B^{\prime}}, \quad \beta \in \operatorname{SL}(2, \mathbb{C}) .
$$

In the obvious way, $\mathscr{S}$ is also an $\mathbb{R}^{+} \otimes \operatorname{SL}(2, \mathbb{C})$ bundle over $\tilde{\mathscr{M}}$, while over $\mathscr{M}$ itself, the structure group consists of those elements of $\mathrm{GL}(2, \mathbb{C})$ with real determinant. Notice that this last group is not connected.

Now consider $\operatorname{SU}(2,2)$ as a bundle over $\tilde{\mathscr{M}}$ with structure group $\mathfrak{W}=\left\{\mathbb{R}^{+} \otimes \operatorname{SL}(2, \mathbb{C})\right\} \cdot \mathfrak{A}$, where $\mathfrak{A}$ is the identity component in $\operatorname{SU}(2,2)$ of the fourparameter abelian group which covers the special conformal transformations. In any representation of $\mathrm{SU}(2,2)$ induced by a representation $\tau$ of $\mathfrak{B}$ with $\mathfrak{A} \subseteq \operatorname{Ker}(\tau)$, the "Mackey functions" 2 will be constant on the cosets of $\mathfrak{U}$ and may be realized as functions on $\mathrm{SU}(2,2) / \mathfrak{A}$. The utility of $\mathscr{S}$ lies precisely in the fact that its elements parametrize this coset space in a convenient way. Each dyad $W_{A^{\prime}}$ labels the coset of $\mathrm{SU}(2,2)$ consisting of all matrices of the form $\left[X, Y,-W_{1^{\prime}}, W_{0^{\prime}}\right]$ (see [4]).

In order to obtain the free fields of helicity $n / 2$, the first step is to induce with the non-unitary representation

$$
\begin{aligned}
& \tau(r, \beta, A): \xi_{A^{\prime} \ldots B^{\prime}} \rightarrow r^{n+2} \bar{\beta}_{A^{\prime}}^{C^{\prime}} \ldots \bar{\beta}_{B^{\prime}}^{D^{\prime}} \xi_{C^{\prime} \ldots D^{\prime}}, \\
& (r, \beta, A) \in\left\{\mathbb{R}^{+} \otimes \operatorname{SL}(2, \mathbb{C})\right\} \cdot \mathfrak{A}
\end{aligned}
$$

on the symmetric subspace of $\otimes^{n}\left(\overline{\mathbb{C}}^{2}\right)$. The resulting functions on $\mathscr{S}$ satisfy the subsidiary condition

$$
\psi_{A^{\prime} \ldots B^{\prime}}\left(r W_{K^{\prime}} \bar{\beta}_{J^{\prime}}^{K^{\prime}}\right)=r^{-n-2} \bar{\beta}_{A^{\prime}}^{C^{\prime}} \ldots \bar{\beta}_{B^{\prime}}^{D^{\prime}} \psi_{C^{\prime} \ldots D^{\prime}}\left(W_{J^{\prime}}\right)
$$

and are thus each homogeneous of total degree $-n-2$ in their arguments. To obtain a solution to the free field Equations (1), it is necessary to impose an

1 We take signatures $(1,-1,-1,-1,-1,1)$ in $\mathbb{R}^{6}$ and $(1,-1,-1,-1)$ in $\mathbb{M}^{4}$. Our twistor conventions are those of Penrose and MacCallum [5], except that we shall not be using abstract indices

2 Cross-sections of the homogeneous vector bundle lifted to the principal bundle. See [7], for example 
additional condition on $\psi$; however, this is not strictly necessary for our purposes, since Equation (6) is sufficient to establish the conformal weight of the fields.

\section{The Grgin Discontinuity}

Now consider $\mathscr{S}$ as a bundle over $\mathscr{M}$. A matrix in the structure group can be written uniquely as either $r \beta$ or $\operatorname{ir} \beta(r>0, \beta \in \mathrm{SL}(2, \mathbb{C}))$, and (6) must be supplemented by the additional relation

$$
\psi\left(i W_{A^{\prime}}\right)=i^{-n-2} \psi\left(W_{A^{\prime}}\right) .^{3}
$$

[We are compelled to choose $\left(6^{\prime}\right)$ rather than, say, $\psi(i W)=i^{-n} \psi(W)$, because $\left(6^{\prime}\right)$ holds automatically for any $\psi$ which is real analytic: any such $\psi$ admits a holomorphic extension to $\mathscr{S}^{*}$ which is complex homogeneous of degree $-n-2$.]

Although $\mathscr{S}$ itself is connected, its fibers over $\mathscr{M}$ are not; this means that the bundle $\mathscr{S} \rightarrow \mathscr{M}$ has no global cross-sections, and this in turn means that $\mathscr{S}$ is not a trivial double cover of a bundle of conformal spin-frames on $\mathscr{M}$. Thus a function such as that displayed in (6) cannot possibly be thought of as an ordinary spinor field on $\mathscr{M}$, even though it has, in a certain sense, the correct local behavior. It should be noted that this holds independently of whether or not $\psi$ satisfies any field equations.

To see the relationship between functions on $\mathscr{S}$ and functions on the usual bundle of conformal spin-frames, note that $\mathscr{S}$ is a four-fold covering of $\mathscr{C}:=C_{0}(1,3) / A$, where $A$ is the subgroup of special conformal transformations. Now $\mathscr{C} \rightarrow \mathscr{M}$ is an $\mathbb{R}^{+} \otimes L_{+}^{\uparrow}$ bundle which does admit a global cross-section: identify $M$ with the $U(2)$ subgroup of $C_{0}(1,3)$, and take the image of this in $\mathscr{C}$. There is another "natural" $\mathbb{R}^{+} \otimes L_{+}^{\uparrow}$ bundle over $\mathscr{M}$-the subbundle $\mathscr{C}^{\prime}$ of the frame bundle consisting of those frames which are orthonormal (and properly oriented) with respect to some metric on $\mathscr{M}$ conformally related to that of Minkowski space. While there is no canonical isomorphism connecting these two bundles, there is an "obvious" one, obtained as follows:

Let $x_{0} \in \mathscr{M}$ be the point representing the origin of Minkowski space, and let $\left[e_{a}\left(x_{0}\right)\right]$ be the standard (Minkowskian) orthonormal tetrad at $x_{0}$. At any other $x \in \mathscr{M}$, define a frame by

$$
\left[e_{a}(x)\right]:=f(x)_{*}\left[e_{a}\left(x_{0}\right)\right],
$$

where $f(x)$ is the unique element of $U(2)$ mapping $x_{0}$ to $x$. Let $F(x)$ be the image of $f(x)$ in $\mathscr{C}$, and identify this with $\left[e_{a}(x)\right]$. This maps a global cross-section of $\mathscr{C}$ onto one of $\mathscr{C}^{\prime}$ and is enough to fix the isomorphism. Note that we can now identify suitable vector-valued functions on $\mathscr{C}$ (obtained by inducing a representation of $C_{0}(1,3)$ with one of $\left.\mathbb{R}^{+} \otimes L_{+}^{\uparrow}\right)$ with appropriate conformally weighted densities on $\mathscr{M}$ (as opposed to just $\mathbb{M}^{+}$). This is analogous to identification of spin and conformally weighted functions with symmetric tensor densities on the two-sphere [8].

Now to see which of the massless free fields can be regarded as spinor fields on $\mathscr{M}$, note that a frame in $\mathscr{C}^{\prime} \cong \mathscr{C}$ is covered by precisely four elements of $\mathscr{S}$. If $W_{A^{\prime}}$, is one such, the others are $\left\{J^{k} W_{A^{\prime}}: k=1,2,3\right\}$ where $J:=i I_{4 \times 4}$ generates the center of $\mathrm{SU}(2,2)$. Consider a set of functions $\psi$ obtained from (6) above. In order that they be

$3 \quad$ This representation clearly extends to $U(1) \otimes \mathbb{R}^{+} \otimes \operatorname{SL}(2, C)$, giving "conformal" $U$-spinors [2] 
consistently identifiable as spinor components on $\mathscr{M}$, it is necessary and sufficient that they be

(a) Single-valued on $\mathscr{C}$ if $n$ is even

(b) Double-valued if $n$ is odd.

But, using $\left(6^{\prime}\right)$,

$$
\psi\left(J W_{A^{\prime}}\right)=i^{-n-2} \psi\left(W_{A^{\prime}}\right)
$$

so that case (b) never occurs, while (a) occurs only for $n \equiv 2(\bmod 4)$, i.e., only in representations of $C_{0}(1,3)$.

If, in spite of this, one tries to interpret $\psi$ as a spinor field on $\mathscr{M}$ for other values of $n$, a spurious discontinuity will arise.

\section{Spinor Fields on $\tilde{\mathscr{M}}$}

We return to thinking of $\mathscr{S}$ as a bundle over $\tilde{\mathscr{M}}$. By repeating the argument above, $\mathscr{S}$ is a double covering of $\tilde{\mathscr{C}}^{\prime}$, the bundle of conformal frames over $\tilde{\mathscr{M}}$, and so a conformal spin structure. Since $\tilde{\mathscr{M}}$ is $S^{1} \times S^{3}, \pi_{1}(\tilde{\mathscr{M}})=\mathbb{Z}$, and there exist precisely two such spin structures; these may be constructed by choosing one of two inequivalent cross-sections of $\tilde{\mathscr{C}}^{\prime}$, so as to decompose it into a product $\tilde{\mathscr{M}} \times\left(\mathbb{R}^{+} \otimes L_{+}^{\uparrow}\right)$, and hence "unwind" it. The structure $\mathscr{S}$ corresponds to choosing the cross-section on a spacelike $S^{3}$-hypersurface and then dragging it round along a congruence of closed null geodesics [2]; the other corresponds to simply dragging round the $S^{1}$ component of $\tilde{\mathscr{M}}$ by time-translation.

Although the second seems to be a more natural spin-structure geometrically, it leads to a Grgin-type discontinuity of $(-1)^{-n-2}$ for the half-integer spin fields we are dealing with here.

As a final remark, we note that the restriction of $\mathscr{S}$ to any simply-connected region of $\tilde{\mathscr{M}}($ or $\mathscr{M})$ is trivial; it is this which allows one to identify a spin-frame at a point of uncompactified Minkowski space with a unique twistor dyad in a consistent fashion $[3,4]$.

Acknowledgement. One of us (C.J.S.C.) records his indebtedness to Dr. P. J. McCarthy for many of the ideas that appear in this paper.

\section{References}

1. Grgin,E.: Ph. D. Thesis, Syracuse University (1966)

2. Clarke, C.J.S.: Commun. math. Phys. 47, 229 (1976)

3. Penrose, R.: Int. J. Theor. Phys. 1, 61 (1968)

4. Lerner,D.: Twistors and induced representations of SU(2, 2). J. Math. Phys. (to appear)

5. Penrose, R., MacCallum,M. A.H. : Phys. Repts. 6C, 242 (1973)

6. Hansen, R., Newman,E.T.: Gen. Rel. Grav. 6, 361 (1975)

7. Herman, R.: Lie groups for physicists. New York: W.A. Benjamin 1966

8. Held,A., Newman,E.T., Posadas, R.: J. Math. Phys. 11, 3145 (1970) 